\title{
SELF-CONSISTENT FIELD THEORY OF A LIPID MONOLAYER
}

\author{
F.W. WIEGEL \\ Department of Applied Physics, Twente University of Technology, Enschede, The Netherlands \\ and
}

\begin{abstract}
A.J. KOX
Institute of Theoretical Physics, University of Amsterdam, Amsterdam, The Netherlands
\end{abstract}

Received 18 July 1978

\begin{abstract}
We present a mean-field theory which is specifically designed to take care of the effects of backfolding in lipid monolayers. Agreement with experiments on DPPC is fair.
\end{abstract}

In a recent paper the authors studied the statistical mechanics of a lipid monolayer using a mathematical analogy with the quantum gas of hard disks [1]. This analogy had been pointed out before by Wiegel in the context of an exactly solvable two-dimensional model [2]. A drawback of this analogy, as well as of most other theories of lipid membranes, consists of the fact that "backfolding" is neglected, i.e. it is assumed that a given lipid always has only one point in common with any plane parallel to the membrane surface. This will be a good approximation at high densities; at intermediate and low densities, however, backfolding will occur and might lead to non-trivial corrections. In this letter we present a theory which is specifically designed to take care of the effects of backfolding. The theory is of the mean-field type, leads to a simpler formalism than most others and is in fair agreement with experiments. A more detailed discussion of this approach will be given in a forthcoming review [3].

The monolayer consists of $M$ identical lipids which are restricted with their head groups to the $x, y$ plane of a cartesian coordinate frame; the hydrocarbon chains are restricted to the half-space $z \geqslant 0$. Each chain is represented by a random walk of $N$ steps, each of length $l$. These random walks will be treated in the continuum approximation; reviews of this method can be found in papers by Freed [4] and Wiegel [5]. On a coarsegrained scale a chain can be described by a continuous trajectory $\boldsymbol{r}(\nu) \equiv(x(\nu), y(\nu), z(\nu))$. For the time being we only consider the hard-core steric repulsion between the chains; a long-range attraction will be added shortly. Suppose a fraction $f(z)$ of space is on the average occupied by polymeric material, i.e. per unit volume only a volume $1-f(z)$ is available for the end points of the steps. The probability that a chain with hinges at $r_{0}, r_{1}, \ldots, r_{N}$ will nowhere overlap with other chains is approximately given by:

$W \approx \prod_{\nu=1}^{N}\left(1-f\left(z_{\nu}\right)\right)$

In the continuum approximation this gives for the weight functional of a chain of configuration $r(\mu)$, $0<\mu<N$ :

$P[r(\mu)] \sim \exp \left\{-\int_{0}^{N}\left[\frac{3}{2 l^{2}}\left(\frac{\mathrm{d} r}{\mathrm{~d} v}\right)^{2}-\ln (1-f(z(\nu)))\right] \mathrm{d} \nu\right\}$.

A term due to the potential energy of the repeating units in the gravitational field has been omitted from eq. (2) because an order of magnitude estimation shows such effects to be negligible.

For reasons of symmetry the most probable configuration of the chain is one in which the trajectory moves perpendicularly to the surface. This configuration will be denoted by $r^{*}(\nu)=\left(0,0, z^{*}(\nu)\right)$ and can 
be found by maximizing the exponential of the last equation. This gives the relation:

$\mathrm{d} z^{*}=\left\{-\frac{2}{3} l^{2} \ln (1-f)\right\}^{1 / 2} \mathrm{~d} \nu$.

The function $f(z)$ can now be determined in a selfconsistent way by the following argument which is similar to an argument used by de Gennes in the theory of self-avoiding random walks [6]. Consider a slice of space of area $\Omega$, with $z$-coordinates between $z^{*}$ and $z^{*}+\mathrm{d} z^{*}$ and with a volume $\Omega \mathrm{d} z^{*}$. If every configuration is approximated by the most probable configuration one finds that $M \mathrm{~d} \nu$ repeating units are contained in this volume $\Omega \mathrm{d} z^{*}$. If every repeating unit excludes an effective volume $\gamma=\frac{4}{3} \pi l^{3}$ for occupancy by the center of any other repeating unit, the excluded fraction of space equals

$f=\gamma \frac{M}{\Omega} \frac{\mathrm{d} \nu}{\mathrm{d} z^{*}}=\gamma \rho\left\{-\frac{2}{3} l^{2} \ln (1-f)\right\}^{-1 / 2}$,

where $\rho=M / \Omega$ denotes the number density of chain molecules in the monolayer. This is an implicit equation which uniquely determines $f$ as a function of $\gamma \rho / l$.

It is now straightforward to calculate the Helmholtz free energy by evaluating the exponential of eq. (2) for the most probable configuration (3). In this way one finds the lateral pressure $\Pi_{0}$ of the system of lipid chains with hard-core repulsions only:

$\Pi_{0}=k_{\mathrm{B}} T\left(\rho+N \rho^{2} \mathrm{~d} \lambda / \mathrm{d} \rho\right)$,

where $\lambda \equiv-2 \ln (1-f)$ and where $k_{\mathrm{B}}$ denotes

Boltzmann's constant and $T$ the temperature.

Long-range attractive forces can be included in the mean-field approximation. This calculation is standard and leads to the equation of state:

$\Pi=k_{\mathrm{B}} T\left(\rho+N \rho^{2} \mathrm{~d} \lambda / \mathrm{d} \rho\right)-\frac{1}{2} w \rho^{2}$

(+ Maxwell's equal area construction),

where $w$ equals the absolute value of the integral of the attractive lipid-lipid interaction over the surface. In terms of the dimensionless variables

$\tilde{\rho}=\rho \frac{\gamma}{l} \sqrt{\frac{3}{2}}, \quad \tilde{\Pi}=\frac{\Pi}{k_{\mathrm{B}} T} \frac{\gamma}{l} \sqrt{\frac{3}{2}}, \quad \tilde{w}=\frac{w}{k_{\mathrm{B}} T} \frac{l}{\gamma} \sqrt{\frac{2}{3}}$,

the equation of state assumes the dimensionless

form:
Table 1

The values of the dimensionless density $\tilde{\rho}_{c}$, pressure $\tilde{\Pi}_{c}$ and interaction energy $\widetilde{w}_{\mathrm{c}}$ at the critical point, as a function of chain length $\mathrm{Nl}$.

\begin{tabular}{|c|c|c|c|}
\hline$N$ & $\tilde{\rho}_{\mathrm{C}}$ & $\tilde{\Pi}_{\mathrm{c}}$ & $\tilde{w}_{\mathrm{c}}$ \\
\hline 10 & 0.305 & 0.235 & 57.7 \\
\hline 11 & 0.301 & 0.243 & 63.1 \\
\hline 12 & 0.297 & 0.252 & 68.6 \\
\hline 13 & 0.294 & 0.260 & 74.0 \\
\hline 14 & 0.292 & 0.269 & 79.4 \\
\hline 15 & 0.289 & 0.280 & 84.9 \\
\hline 16 & 0.287 & 0.286 & 90.3 \\
\hline 17 & 0.286 & 0.296 & 95.7 \\
\hline 18 & 0.284 & 0.305 & 101 \\
\hline 19 & 0.283 & 0.314 & 107 \\
\hline 20 & 0.281 & 0.323 & 112 \\
\hline 21 & 0.280 & 0.332 & 117 \\
\hline 22 & 0.279 & 0.341 & 123 \\
\hline 23 & 0.278 & 0.350 & 128 \\
\hline 24 & 0.277 & 0.359 & 134 \\
\hline 25 & 0.276 & 0.368 & 139 \\
\hline 26 & 0.275 & 0.380 & 145 \\
\hline 27 & 0.274 & 0.387 & 150 \\
\hline 28 & 0.274 & 0.397 & 155 \\
\hline 29 & 0.273 & 0.406 & 161 \\
\hline 30 & 0.272 & 0.415 & 166 \\
\hline 31 & 0.272 & 0.424 & 172 \\
\hline 32 & 0.271 & 0.433 & 177 \\
\hline 33 & 0.271 & 0.442 & 182 \\
\hline 34 & 0.270 & 0.453 & 188 \\
\hline 35 & 0.270 & 0.462 & 193 \\
\hline 36 & 0.269 & 0.471 & 199 \\
\hline 37 & 0.269 & 0.480 & 204 \\
\hline 38 & 0.268 & 0.490 & 209 \\
\hline 39 & 0.268 & 0.500 & 215 \\
\hline 40 & 0.268 & 0.508 & 220 \\
\hline
\end{tabular}

$\tilde{\Pi}=\tilde{\rho}+N \tilde{\rho}^{2} \mathrm{~d} \lambda / \mathrm{d} \tilde{\rho}-\frac{1}{2} \tilde{w} \tilde{\rho}^{2}$

(+ Maxwell's equal area construction),

$\lambda(\tilde{\rho})=-2 \ln (1-f), \quad \tilde{\rho}^{2}=-f^{2} \ln (1-f)$.

This expression for the lateral pressure of the monolayer predicts a critical temperature below which coexisting phases appear. The critical point occurs for that density $\tilde{\rho}_{\mathrm{c}}$ for which $\mathrm{d} \tilde{\Pi} / \mathrm{d} \tilde{\rho}=0$ and $\mathrm{d}^{2} \tilde{\Pi} / \mathrm{d} \tilde{\rho}^{2}=0$. For a given value of $N$ this implies a specific value $\widetilde{w}_{\mathrm{c}}$ of $\tilde{w}$. We have numerically calculated $\tilde{\rho}_{\mathrm{c}}, \widetilde{\Pi}_{\mathrm{c}}$ and $\tilde{w}_{\mathrm{c}}$ as functions of the chain length $N l$; the results are given in table 1 .

Experiments on lipid monolayers show a phase tran- 
tion indeed, but the observed isotherms are not flat in the coexistence region; this might especially be due to the metastable nature of the high density phase [7]. Nagle [8] has estimated a critical point for dipalmitoyl phosphatidyl choline (DPPC): $T_{\mathrm{c}} \approx 305 \mathrm{~K} ; \Pi_{\mathrm{c}} \approx 25$ $\mathrm{dyn} / \mathrm{cm} ; \rho_{\mathrm{c}}^{-1} \approx 26.5 \times 10^{-16} \mathrm{~cm}^{2} / \mathrm{chain}$. Using these values for the critical point to fit the critical isotherm one finds that at the critical point about $39 \%$ of space is blocked by polymeric material: $N=31, l \approx 1.2 \AA$ and $w \approx 0.52 \times 10^{-26} \mathrm{~cm}^{2} \mathrm{erg}$. These figures are all of the correct order of magnitude [9].

The work of one of us (A.J.K.) is part of the research programme of the "Stichting voor fundamenteel onderzoek der materie" (FOM), which is financially sup- ported by the "Nederlandse organisatie voor zuiver wetenschappelijk onderzoek" (ZWO).

References

[1] A.J. Kox and F.W. Wiegel, Statistical mechanics of a lipid monolayer, Physica, to be published.

[2] F.W. Wiegel, J. Stat. Phys. 13 (1975) 515.

[3] F.W. Wiegel, and A.J. Kox, Theories of lipid monolayers, Adv. Chem. Phys., to be published.

[4] K. Freed, Adv. Chem. Phys. 22 (1972) 1.

[5] F.W. Wiegel, Phys. Rep. 16 (1975) 57.

[6] P.G. de Gennes, Rep. Prog. Phys. 32 (1969) 187.

[7] L.W. Horn and N.L. Gershfeld, Biophys. J. 18 (1977) 301.

[8] J.F. Nagle, J. Membr. Biol. 27 (1976) 233.

[9] J.F. Nagle, J. Chem. Phys. 63 (1975) 1255. 